\title{
Nuclear Odontogenic Ameloblast-Associated Protein (ODAM) Correlates with Melanoma Sentinel Lymph Node Metastasis
}

\author{
Sagar S. Gandhi, ${ }^{1,2}$, Daniel P. Kestler, ${ }^{2,3}$, Charles T. Bruker ${ }^{4}$, James M. McLaughlin ${ }^{1,2}$, Robert E. Heidel ${ }^{2}$, \\ Sabina Siddiqui ${ }^{5}$, James S. Foster ${ }^{2,3}$, Keith D. Gray ${ }^{1,2}$, John Bell ${ }^{1,2}$, Alan Solomon ${ }^{2,3}$, James Lewis ${ }^{1,2}$ \\ ${ }^{1}$ Department of Surgery, University of Tennessee Medical Center-Knoxville, Knoxville, USA; ${ }^{2}$ Graduate School of Medicine, Uni- \\ versity of Tennessee Medical Center-Knoxville, Knoxville, USA; ${ }^{3}$ Department of Medicine, University of Tennessee Medical Cen- \\ ter-Knoxville, Knoxville, USA; ${ }^{4}$ Department of Pathology, Boca Raton Regional Hospital, Boca Raton, USA; ${ }^{5}$ Department of Sur- \\ gery, University of Michigan School of Medicine, Ann Arbor, USA.
}

Email: dkestler@utmck.edu

Received August $8^{\text {th }}, 2013$; revised September $2^{\text {nd }}, 2013$; accepted September $9^{\text {th }}, 2013$

Copyright (C) 2013 Sagar S. Gandhi et al. This is an open access article distributed under the Creative Commons Attribution License, which permits unrestricted use, distribution, and reproduction in any medium, provided the original work is properly cited.

\begin{abstract}
We have examined primary tumor sections from melanoma patients by immunohistochmistry (IHC) for the presence of the odontogenic ameloblast-associated protein (ODAM). Within these patient tissues we have observed a correlation of nuclear ODAM staining in the primary tumors with sentinel lymph node (SLN) metastasis. Surgically, SLN invasion in melanoma is considered an important indicator of more aggressive, invasive melanoma and to date there are limited biomarkers which strongly correlate with metastatic disease. The observation that ODAM staining in melanoma associates with SLN invasion may have important prognostic implications which could assist in the management of melanoma. Notably, ODAM expression may correlate with pathway-signaling we have previously reported to be affected by ectopic ODAM expression in cultured melanoma and breast cancer cell lines.
\end{abstract}

Keywords: Melanoma; Sentinel Lymph Node Metastases; ODAM; Immunohistochemistry

\section{Introduction}

Melanoma metastasis is predicted by factors that reflect biologic behavior such as primary tumor Breslow thickness, mitotic rate, and ulceration [1,2]. Sentinel lymph node (SLN) status in melanoma remains the single most important predictor of overall survival [3-5]. In addition, records from the AJCC Melanoma Staging Database demonstrate that as Breslow thickness increases, a significant decline in both 5- and 10-year survival rates is observed, and recent data demonstrate a significant correlation between survival and the primary tumor mitotic rate. Notably, survival rates of patients with an ulcerated melanoma and similar Breslow thickness are significantly worse compared to non-ulcerated matched primary tumors [2]. Many potential biomarkers for melanoma have been reported, but their clinical significance largely remains undetermined [6]. Molecular factors influencing primary melanoma growth and metastasis reflect dysregulation of normal cellular signaling pathways, and these factors continue to be intensively investigated, both with respect to potential therapeutic advances and for utility as prognostic indicators [7].

ODAM is a protein initially identified as the amyloidforming component in a rare dental neoplasm, calcifying epithelial odontogenic tumor or Pindborg tumor. The protein has been detected in a broad range of epithelial tissues and in multiple human cancers including those of the breast, esophagus, gastric tissues, and bronchial epithelium $[8,9]$. The potential role of this protein as a marker of disease status and survival in breast cancer has been reported as increased nuclear ODAM staining of primary breast tumors across disease staging [10]. A portion of patients (up to $\sim 13 \%$ in late stage) who were ODAM-positive exhibited improved survival compared to stage-matched ODAM-negative breast cancer patients while the remaining bulk ( $87 \%)$ of late stage ODAMpositive tumor patients did not exhibit a survival benefit, suggesting at least two patients' outcome groups associated with ODAM-expressing breast tumors [10]. Based on previous observations that lymph node-positive breast 
cancer patients are often positive for nuclear ODAM staining, together with the propensity of melanomas to metastasize into regional lymph nodes, we examined ODAM expression in primary tumors and lymph node biopsies of patients with SLN-positive (Stage III) and SLN-negative (Stage I-II) melanoma. This allowed us to test whether nuclear ODAM staining in primary melanoma could predict sentinel node positivity. We report our findings as follows.

\section{Materials and Methods}

\subsection{Melanoma Patient Tumor Tissue}

Patients diagnosed with melanoma were identified retrospectively through our institutional tumor registry from years 2000-2006. Only cases with available primary tumor and SLN tissue were evaluated. Histological features of the primary tumors were recorded for both the SLNnegative and positive samples, along with overall survival (OS) and recurrence data obtained from our patient database. Archived formalin-fixed paraffin embedded tumor tissues were cut and immunostained with murine monoclonal anti-ODAM antibody $8 \mathrm{~B} 4$, as previously reported [10] and detailed below.

\subsection{IHC Analysis}

Two micrometer thick formalin-fixed paraffin embedded melanoma sections were mounted on charged slides (Fisherbrand Superfrost/Plus, Thermo-Fisher), dried overnight at room temperature, and deparaffinized to water. Sections were immersed in antigen-retrieval solution (Biogenex Citra Plus, BioGenex, San Ramon, CA, USA) and subjected to standard blocking procedures. AntiODAM antibody was applied at 1:7500 in diluent (Dako, \#S-3022), incubated overnight $\left(5^{\circ} \mathrm{C}\right)$, and visualized using the ImmPRESS polymerized enzyme-linked reporter system followed by the ImmPACT diaminobenzidine detection kit (Vector Laboratories, Burlingame, CA, USA). External positive controls were utilized for slide interpretation with each batch of patient tumor-slides while, benign structures present in each study section served as internal controls.

Tumor tissue obtained from each block was stained by hematoxylin-eosin and reviewed in conjunction with immunostained slides. The presence or absence of ODAM immunostaining was determined in the neoplastic cells of each case and staining was assessed and reported, for the nucleus only, as negative or positive. Nuclear positivity was defined by the presence of distinct smooth homogenous staining of at least $50 \%$ of tumor cell nuclei, and negativity was defined as a near complete lack of nuclear immunostaining in essentially all tumor cells of interest (less than $1 \%$ of nuclei) as in previous studies [10]. This was based on the observed distribution of nuclear ODAM staining where positive tumors exhibited staining in essentially all (greater than $90 \%$ ) cell nuclei while staining was present in less than $1 \%$ of cell nuclei in tumors designated as ODAM-negative. Thus, tumors with a percent reactivity between $1 \%$ and $90 \%$ were rare, and $50 \%$ reactivity was chosen as a cutoff to provide a study design that minimized ambiguity. In practice, no tumors exhibited reactivity near the 50\%-positive cutoff. Staining for ODAM was graded in blinded fashion by a single peer reviewed pathologist (CTB).

\subsection{Statistics}

Descriptive statistics were conducted for the Breslow thickness, age, gender, and months to follow-up. An independent samples t-test was used to compare Breslow values on SLN-positive and SLN-negative groups. In the event of a violation of a statistical assumption, a nonparametric Mann-Whitney U test was employed. Unadjusted odds ratios (OR) with 95\% confidence intervals (CI) were calculated to compare ODAM staining and SLN status to various discrete variables including recurrence, cancer status, and cancer death. Logistic regression analysis was employed to yield multivariate adjusted odds ratios when predicting for SLN positivity. Statistical significance was assumed at a $p<0.05$ level and all analyses were conducted using SPSS Version 19 software (SPSS, Chicago, IL).

\section{Results}

\subsection{Patient Sample Populations}

Our institutional tumor registry contained 270 cases of primary melanoma treated from 2000-2006. Complete data and adequate tissue samples were available for 44 of these patients (21 SLN-positive patients and 23 SLNnegative patients). Inadequate tissue samples, incomplete medical records, non-sentinel lymph node biopsies and patients who underwent lymphadenectomies were excluded. All patients had invasive melanoma. Table 1 depicts patient demographics and primary tumor characteristics for each cohort. Demographics were not significantly different but was close to significance in regards to Breslow thickness $(p=0.09)$. Ulceration was not significantly different between cohorts, 13 versus 10 in the SLN-positive and SLN-negative cohorts, respectively ( $p$ $=0.61$ ).

\subsection{ODAM Immunostaining}

Immunostaining for ODAM showed that both the nucleus and cytoplasm of benign melanocytes stain strongly positive for ODAM, while in melanoma, staining of the 
with Melanoma Sentinel Lymph Node Metastasis

Table 1. Melanoma patient characteristics ${ }^{\mathrm{a}}$.

\begin{tabular}{|c|c|c|c|c|}
\hline & SLN-NEGATIVE & SLN-POSITIVE & ODAM-NEGATIVE & ODAM-POSITIVE \\
\hline AGE (Median) & $68(44-85)$ & $54(34-75)$ & $68(34-85)$ & $56(39-80)$ \\
\hline GENDER (M:F) & $10: 13$ & $12: 9$ & $8: 15$ & $14: 7$ \\
\hline \multicolumn{5}{|l|}{ LOCATION } \\
\hline Extremities & 14 & 8 & 13 & 9 \\
\hline Trunk & 4 & 9 & 7 & 6 \\
\hline Head & 5 & 4 & 3 & 6 \\
\hline MEDIAN BRESLOW (Range) & $2 \mathrm{~mm}(0.35-7)$ & $3.38 \mathrm{~mm}(0.32-16)$ & $2.48 \mathrm{~mm}(0.35-10)$ & $2.75 \mathrm{~mm}(0.32-16)$ \\
\hline ULCERATION PRESENT & 10 & 13 & 13 & 10 \\
\hline
\end{tabular}

${ }^{a}$ Cohort Demographics are separated by sentinel lymph node-negative (Stage I/II), sentinel lymph node-positive (Stage III), ODAM-negative, and ODAMpositive melanoma.

cytoplasm is consistently diminished and nuclear staining is variable. In ODAM-negative melanoma there is no nuclear staining of the primary tumor (Figure 1), while ODAM-positive melanoma exhibits readily demonstrable staining in the cell nucleus (Figure 2). Primary tumors in the SLN-positive cohort were significantly more likely to exhibit nuclear localization of ODAM as recorded in Table 2. Sixteen of 21 specimens (76\%) in the SLNpositive patients stained for ODAM compared to 5 of 23 $(22 \%)$ in the SLN-negative patients (Odds Ratio (OR) = $11.52,95 \%$ CI $2.81,47.23)$. SLN staining for ODAM corresponded with the primary tissue staining pattern in all specimens. No SLN stained positive for ODAM unless the primary tumor was ODAM-positive as well. Our study had 13 thin melanomas $(\leq 1 \mathrm{~mm}), 5$ of which $(38 \%)$ were ultimately Stage III. Notably, of these five, four stained positive for ODAM. Also, 4 of 13 thin melanomas were ulcerated; 1 of these was SLN-negative, 3 were SLN-positive, and ODAM status correlated with SLN status in all samples from ulcerative tumors.

\subsection{Recurrence and Survival Analyses}

Median follow-up for the SLN-positive and negative cohorts was 37 months (range of 7 - 68) and 47 months (range of 4 -64), respectively. Four patients were lost to follow-up, all of which were in the SLN-negative group (half were ODAM-positive). Logistic regression analysis, given in Table 3, found that when controlled for Breslow thickness and ulceration, participants that stained ODAMpositive were 35 times more likely to be SLN-positive $(\mathrm{OR}=35,95 \%$ CI 4.05 - 302.26). Breslow thickness was close to showing a significant association (OR 1.48, 95\% CI $0.96-2.27, \mathrm{p}=0.077$ ).

As shown in Table 4, disease recurrence developed in 10 of $18(55.5 \%)$ node positive patients and 2 of 22 (9.1\%) node negative patients ( $\mathrm{OR}=12.5,95 \%$ CI 2.23 ,
70.19). Three patients in the SLN-positive group were found to have metastatic disease during staging after initial wide local excision and SLN biopsy and were thus considered never disease free. Subset analysis of ODAMpositive patients irrespective of nodal status demonstrated that 10 of $20(50 \%)$ ODAM-positive patients had recurrence versus 2 of $20(10 \%)$ in the ODAM-negative group $(\mathrm{OR}=9,95 \%$ CI $1.64,49.45)$. Overall survival (OS) was 10 of $21(48 \%)$ in the SLN-positive group and 18 of $23(79 \%)$ in the SLN-negative group (3.96, 95\% CI $1.07,14.67)$. OS in the ODAM-positive group was 11 of $21(52 \%)$ versus 17 of $23(74 \%)$ for ODAM-negative primary tumors $(\mathrm{OR}=0.39,95 \%$ CI $0.11,1.38)$ as displayed in Table 5.

\section{Discussion}

Our study revealed that SLN-positive primary tumors (Stage III) were significantly more likely to exhibit nuclear ODAM upon IHC staining, and thus suggests that nuclear ODAM localization is associated with more invasive tumors. Furthermore, 4 of 5 SLN-positive thin melanomas $(<1 \mathrm{~mm})$ were found to be nuclear ODAMpositive. Since an estimated $5 \%-8 \%$ of thin melanomas metastasize to lymph nodes, SLN biopsies are not routinely performed in these cases [5]. We propose that preoperative staining of melanoma, and particularly thin melanoma, for ODAM may guide operative management and patient treatment.

ODAM is expressed in a broad range of normal epithelial tissues and malignancies [8,9]. During dental development the protein is secreted from ameloblasts and associated with the junctional epithelium at the incisor enamel interface [11,12]. Late in dental development ODAM localizes to the ameloblast nucleus and mediates direct activation of MMP-20/enamelysin gene expression [13]. Thus, while its roles have not been fully delineated, 

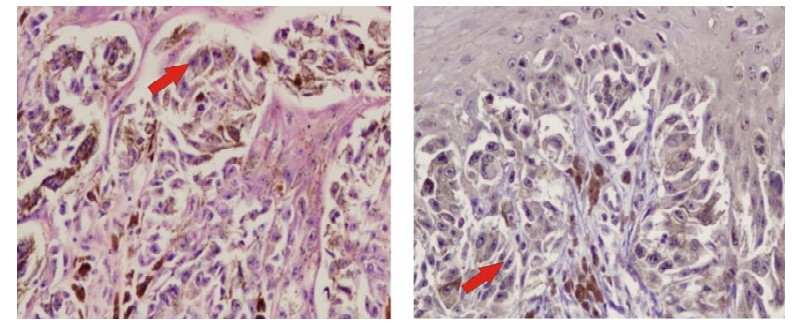

Figure 1. ODAM-negative primary melanoma (stage I). Left: hematoxylin-eosin stain. Right: anti-ODAM immunostain. Arrows indicate tumor cell nuclei. Note brown staining of phagocytic cells with lack of nuclear staining in tumor cells. Original magnifications $200 \times$.

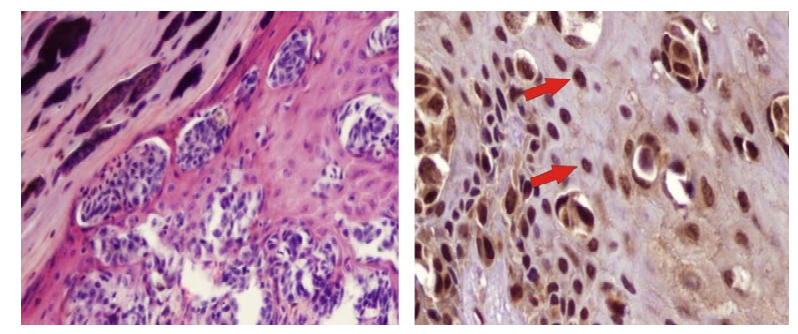

Figure 2. ODAM-positive primary melanoma (stage III). Left: hematoxylin-eosin stain (original magnification 100×). Right: anti-ODAM immunostain (original magnification $200 \times$ ). The arrows indicate two of numerous ODAM-positive tumor cell nuclei.

ODAM exhibits potential cell signaling functions both in the nucleus and through interactions with extracellular matrix components, suggesting classification of ODAM as a matricellular protein [12-14]. Proteins of this subset include the tenascin, osteopontin, thrombospondin, SPARC (osteonectin), SPARCL1 (Hevin), and CCN proteins. This class of proteins has been suggested to contribute to melanoma progression by supporting cellular release from keratinocyte control [15].

The high degree of cellular organization of normal differentiated tissues is often lost in cancer. The detection of ODAM nuclear localization in melanoma is consistent with the observation of other proteins, such as activating transcription factor 2 (ATF2), showing increased localization to the nucleus in metastatic melanoma [16]. Nuclear re-localization of forkhead box 03 (FOXO3a), $\beta$ catenin, and a number of other proteins is proving to be a determining factor in tumor cell growth and invasiveness associated with a broad range of malignancies including melanoma [17-19].

As of yet, no singular molecular biomarkers have proven clinical utility for predicting the progression of melanoma to metastasis [20-22]. Previous studies have discussed biomarkers such as S100B and lactate dehydrogenase as prognostic indicators for melanomas [6,20-24]. These biomarkers are elevated in advanced disease, and their presence indicates poor prognosis and diminished survival. However, they are not routinely used in clinical practice for early stage disease. Similarly, markers for lymphatic vessel density have been utilized as predictors of SLN metastasis, but this requires analysis of multiple antigens by IHC and a high degree of variability has been reported [25]. Recent reports have also described two potential biomarkers for melanoma identified by monoclonal antibodies KBAb2 and PNL2 and observed in over $85 \%$ of cases but the associated antigens have not, to date, been identified [26].

A previous retrospective study of breast cancer tumor sections at our institution demonstrated a statistically significant correlation between the presence of nuclear ODAM and tumor stage [10]. Our current study also demonstrates that ODAM expression correlates with melanoma recurrence, survival, and that Breslow thickness approached significance when associated with ODAM staining.

These observations underlie recent research in our laboratory which demonstrated profound growth inhibition in vitro and in vivo with human breast cancer cells engineered to express ODAM. This corresponds with suppression of the PI3K/AKT/mTOR signaling pathway $[27,28]$. Notably, melanoma exhibits frequent dysregulation of the PI3K/AKT/mTOR and RAS/RAF/MAPK signaling pathways $[7,29,30]$ and we have observed growth suppression in vitro and AKT inhibition upon ectopic ODAM expression in human melanoma cell lines [28]. This suggests potential impacts on tumor cell behavior, disease progression, and therapeutic outcomes when ODAM is expressed in these malignancies. Yet, in the presence of functional, or dysregulated signaling pathway components, these effects may differ. Our studies thus serve to highlight the complex interactions between signaling pathways in melanoma, given that single drug inhibitors can yield contrary effects on tumor behavior dependent upon the cellular context [31-34].

Recognizing the complexity of ODAM function with respect to cellular localization, and the participation of multiple signaling pathways in driving melanoma growth, clarification of the role of ODAM expression in melanoma biology is necessary both in mechanistic terms, and as support for any possible clinical utility of staining for ODAM. The study described herein was of moderate size and retrospective in nature. Thus, the correlation of ODAM expression with SLN positivity and overall survival will be better delineated by analyses comprised of greater sample size and longer follow-up intervals, which will allow for increased statistical power in the results. Further, a clear assessment of the association of ODAM with melanoma spread will also depend on studies which place ODAM expression in the context of evolving subtype classifications for melanoma [35] and the associated molecular hallmarks of these subtypes (e.g.; BRAF V600E, c-KIT, NRAS, cyclin D). 
Table 2. Distribution of ODAM staining of primary tumor tissue $\mathrm{e}^{\mathrm{a}}$.

\begin{tabular}{cccc}
\hline & ODAM-POSITIVE $(\mathbf{n}=\mathbf{2 1})$ & ODAM-NEGATIVE $(\mathbf{n}=\mathbf{2 3})$ & ODDS RATIO/CI \\
\hline SLN-POSITIVE & 16 & 5 & $11.52 / 95 \% \mathrm{CI}(2.81,47.23)$ \\
SLN-NEGATIVE & 5 & 18 & \\
\hline
\end{tabular}

${ }^{a}$ ODAM staining of primary tumor tissue for lymph node-positive melanoma (Stage III) and lymph node-negative melanoma (Stage I/II).

Table 3. Logistic regression analysis results for ODAM staining, Breslow thickness values, and ulceration of primary melanomas.

\begin{tabular}{cccc}
\hline & ODDS RATIO & $\mathbf{9 5 \% ~ C I}$ & p-value \\
\hline ODAM & 35 & $(4.05,302.3)$ & 0.001 \\
BRESLOW & 1.48 & $(0.96,2.27)$ & 0.07 \\
ULCERATION & 2.08 & $(0.31,14.16)$ & 0.46 \\
\hline
\end{tabular}

Table 4. Recurrence rates of melanoma patients subdivided by sentinel lymph node and ODAM status.

\begin{tabular}{ccccc}
\hline & RECURRENCE & NO RECURRENCE & NEVER DISEASE FREE & ODDS, RATIO/CI \\
\hline SLN-POSITIVE & 10 & 8 & 3 & $12.5 / 95 \% \mathrm{CI}(2.23,70.19)$ \\
SLN-NEGATIVE & 2 & 20 & 1 & $9.0 / 95 \% \mathrm{CI}(1.64,49.45)$ \\
ODAM-POSITIVE & 10 & 10 & 1 & 3 \\
ODAM-NEGATIVE & 2 & 18 & 3 \\
\hline
\end{tabular}

Table 5. Overall survival of melanoma patients subdivided by sentinel lymph node and ODAM status.

\begin{tabular}{cccc}
\hline & ALIVE & MEDIAN FOLLOW-UP (months) & ODDS RATIO/CI \\
\hline SLN-POSITIVE & 10 & 37 & $3.96 / 95 \% \mathrm{CI}(1.07,14.67)$ \\
SLN-NEGATIVE & 18 & 47 & $0.39 / 95 \% \mathrm{CI}(0.11,1.38)$ \\
ODAM-POSITIVE & 11 & 38 & 46 \\
ODAM-NEGATIVE & 17 & 48 \\
\hline
\end{tabular}

\section{Conclusion}

In summary, our results indicate that nuclear localization of ODAM in primary melanoma specimens corresponds with an increased likelihood of SLN metastasis and as such may have important prognostic implications. Further, this correlation may foster an understanding of the complex genetics, host-interaction and signaling pathways involved with both nodal and distant metastases in melanoma. Future studies will require analysis of additional specimens from melanoma or other malignancies exhibiting ODAM expression, with an aim of further correlating ODAM expression with disease characteristics, and its possible relationship to known regulatory biomarkers.

\section{Acknowledgements}

This Research was supported in part through grants from the Susan G. Komen Foundation (DPK) and the University of Tennessee Medical Center Physicians Medical Education Research Foundation (JL). The authors also wish to acknowledge Sallie Macy for her efforts towards the immunohistochemistry studies.

\section{REFERENCES}

[1] W. H. Clark Jr., D. E. Elder, D. T. Guerry, L. E. Braitman, B. J. Trock, D. Schultz, M. Synnestvedt and A. C. Halpern, "Model Predicting Survival in Stage I Melanoma Based on Tumor Progression," Journal of the National Cancer Institute, Vol. 81, No. 24, 1989, pp. 18931904. http://dx.doi.org/10.1093/jnci/81.24.1893

[2] C. M. Balch, J. E. Gershenwald, S. J. Soong, J. F. Thompson, M. B. Atkins, D. R. Byrd, A. C. Buzaid, A. J. Cochran, D. G. Coit, S. Ding, A. M. Eggermont, K. T. Flaherty, P. A. Gimotty, J. M. Kirkwood, K. M. McMas- 
ters, M. C. Mihm Jr., D. L. Morton, M. I. Ross, A. J. Sober and V. K. Sondak, "Final Version of 2009 AJCC Melanoma Staging and Classification," Journal of Clinical Oncology, Vol. 27, No. 36, 2009, pp. 6199-6206. http://dx.doi.org/10.1200/JCO.2009.23.4799

[3] F. Amersi and D. L. Morton, "The Role of Sentinel Lymph Node Biopsy in the Management of Melanoma," Advances in Surgery, Vol. 41, 2007, pp. 241-256. http://dx.doi.org/10.1016/i.yasu.2007.05.015

[4] D. L. Rousseau, Jr., M. I. Ross, M. M. Johnson, V. G. Prieto, J. E. Lee, P. F. Mansfield and J. E. Gershenwald, "Revised American Joint Committee on Cancer Staging Criteria Accurately Predict Sentinel Lymph Node Positivity in Clinically Node-Negative Melanoma Patients," Annals of Surgical Oncology, Vol. 10, No. 5, 2003, pp. 569574. http://dx.doi.org/10.1245/ASO.2003.09.016

[5] D. L. Morton, J. F. Thompson, R. Essner, R. Elashoff, S. L. Stern, O. E. Nieweg, D. F. Roses, C. P. Karakousis, N. Mozzillo, D. Reintgen, H. J. Wang, E. C. Glass and A. J. Cochran, "Validation of the Accuracy of Intraoperative Lymphatic Mapping and Sentinel Lymphadenectomy for Early-Stage Melanoma: A Multicenter Trial. Multicenter Selective Lymphadenectomy Trial Group," Annals of Surgery, Vol. 230, No. 4, 1999, pp. 453-463, Discussion $463-455$.

[6] B. E. Gould Rothberg, M. B. Bracken and D. L. Rimm, "Tissue Biomarkers for Prognosis in Cutaneous Melanoma: A Systematic Review and Meta-Analysis," Journal of the National Cancer Institute, Vol. 101, No. 7, 2009, pp. 452-474. http://dx.doi.org/10.1093/jnci/djp038

[7] I. Yajima, M. Y. Kumasaka, N. D. Thang, Y. Goto, K. Takeda, O. Yamanoshita, M. Iida, N. Ohgami, H. Tamura, Y. Kawamoto and M. Kato, "RAS/RAF/MEK/ERK and PI3K/PTEN/AKT Signaling in Malignant Melanoma Progression and Therapy," Dermatology Research and Practice, Vol. 2012, 2012, Article ID: 354191. http://dx.doi.org/10.1155/2012/354191

[8] A. Solomon, C. L. Murphy, K. Weaver, D. T. Weiss, R. Hrncic, M. Eulitz, R. L. Donnell, K. Sletten, G. Westermark and P. Westermark, "Calcifying Epithelial Odontogenic (Pindborg) Tumor-Associated Amyloid Consists of a Novel Human Protein," Journal of Laboratory and Clinical Medicine, Vol. 142, No. 5, 2003, pp. 348-355. http://dx.doi.org/10.1016/S0022-2143(03)00149-5

[9] D. P. Kestler, J. S. Foster, S. D. Macy, C. L. Murphy, D. T. Weiss and A. Solomon, "Expression of Odontogenic Ameloblast-Associated Protein (ODAM) in Dental and Other Epithelial Neoplasms," Molecular Medicine, Vol. 14, No. 5-6, 2008, pp. 318-326. http://dx.doi.org/10.2119/2008-00010.Kestler

[10] S. Siddiqui, C. T. Bruker, D. P. Kestler, J. S. Foster, K. D. Gray, A. Solomon and J. L. Bell, "Odontogenic Ameloblast Associated Protein as a Novel Biomarker for Human Breast Cancer," The American Surgeon, Vol. 75, No. 9, 2009, pp. 769-775.

[11] J. C. Park, J. T. Park, H. H. Son, H. J. Kim, M. J. Jeong, C. S. Lee, R. Dey and M. I. Cho, "The Amyloid Protein APin is Highly Expressed during Enamel Mineralization and Maturation in Rat Incisors," European Journal of
Oral Sciences, Vol. 115, No. 2, 2007, pp. 153-160. http://dx.doi.org/10.1111/j.1600-0722.2007.00435.x

[12] P. Moffatt, C. E. Smith, R. St-Arnaud and A. Nanci, "Characterization of Apin, a Secreted Protein Highly Expressed in Tooth-Associated Epithelia," Journal of Cellular Biochemistry, Vol. 103, No. 3, 2008, pp. 941-956. http://dx.doi.org/10.1002/jcb.21465

[13] H. K. Lee, D. S. Lee, H. M. Ryoo, J. T. Park, S. J. Park, H. S. Bae, M. I. Cho and J. C. Park, "The Odontogenic Ameloblast-Associated Protein (ODAM) Cooperates with RUNX2 and Modulates Enamel Mineralization via Regulation of MMP-20," Journal of Cellular Biochemistry, Vol. 111, No. 3, 2010, pp. 755-767. http://dx.doi.org/10.1002/jcb.22766

[14] C. Nishio, R. Wazen, S. Kuroda, P. Moffatt and A. Nanci, "Expression Pattern of Odontogenic Ameloblast-Associated and Amelotin during Formation and Regeneration of the Junctional Epithelium," European Cells and Materials, Vol. 20, 2010, pp. 393-402.

[15] M. Fukunaga-Kalabis, A. Santiago-Walker and M. Herlyn, "Matricellular Proteins Produced by Melanocytes and Melanomas: In Search for Functions," Cancer Microenvironment, Vol. 1, No. 1, 2008, pp. 93-102. http://dx.doi.org/10.1007/s12307-008-0009-0

[16] A. J. Berger, H. M. Kluger, N. Li, E. Kielhorn, R. Halaban, Z. Ronai and D. L. Rimm, "Subcellular Localization of Activating Transcription Factor 2 in Melanoma Specimens Predicts Patient Survival," Cancer Research, Vol. 63, No. 23, 2003, pp. 8103-8107.

[17] R. G. Bedolla, Y. Wang, A. Asuncion, K. Chamie, S. Siddiqui, M. M. Mudryj, T. J. Prihoda, J. Siddiqui, A. M. Chinnaiyan, R. Mehra, R. W. de Vere White and P. M. Ghosh, "Nuclear versus Cytoplasmic Localization of Filamin A in Prostate Cancer: Immunohistochemical Correlation with Metastases," Clinical Cancer Research, Vol. 15, No. 3, 2009, pp. 788-796. http://dx.doi.org/10.1158/1078-0432.CCR-08-1402

[18] J. J. Derry, G. S. Prins, V. Ray and A. L. Tyner, "Altered Localization and Activity of the Intracellular Tyrosine Kinase BRK/Sik in Prostate Tumor Cells," Oncogene, Vol. 22, No. 27, 2003, pp. 4212-4220.

http://dx.doi.org/10.1038/sj.onc. 1206465

[19] S. P. Tenbaum, P. Ordonez-Moran, I. Puig, I. Chicote, O. Arques, S. Landolfi, Y. Fernandez, J. R. Herance, J. D. Gispert, L. Mendizabal, S. Aguilar, S. Ramon y Cajal, S. Schwartz, Jr., A. Vivancos, E. Espin, S. Rojas, J. Baselga, J. Tabernero, A. Munoz and H. G. Palmer, "Beta-Catenin Confers Resistance to PI3K and AKT Inhibitors and Subverts FOXO3a to Promote Metastasis in Colon Cancer," Nature Medicine, Vol. 18, No. 6, 2012, pp. 892-901. http://dx.doi.org/10.1038/nm.2772

[20] N. K. Haass and K. S. Smalley, "Melanoma Biomarkers: Current Status and Utility in Diagnosis, Prognosis, and Response to Therapy," Molecular Diagnosis and Therapy, Vol. 13, No. 5, 2009, pp. 283-296. http://dx.doi.org/10.1007/BF03256334

[21] A. R. Larson, E. Konat and R. M. Alani, "Melanoma Biomarkers: Current Status and Vision for the Future," Nature Clinical Practice Oncology, Vol. 6, No. 2, 2009, 
pp. 105-117. http://dx.doi.org/10.1038/ncponc1296

[22] C. W. Joyce, I. G. Murphy, M. Rafferty, D. Ryan, E. W. McDermott and W. M. Gallagher, "Tumor Profiling Using Protein Biomarker Panels in Malignant Melanoma: Application of Tissue Microarrays and Beyond," Expert Review of Proteomics, Vol. 9, No. 4, 2012, pp. 415-423. http://dx.doi.org/10.1586/epr.12.5

[23] T. S. Wang, T. M. Johnson, P. N. Cascade, B. G. Redman, V. K. Sondak and J. L. Schwartz, "Evaluation of Staging Chest Radiographs and Serum Lactate Dehydrogenase for Localized Melanoma," Journal of the American Academy of Dermatology, Vol. 51, No. 3, 2004, pp. 399-405. http://dx.doi.org/10.1016/j.jaad.2004.02.017

[24] U. Bottoni, P. Izzo, A. Richetta, T. J. Mannooranparampil, V. Devirgiliis, M. Del Giudice, M. G. Reale, L. Frati and S. Calvieri, "S100 Serum Level: A Tumour Marker for Metastatic Melanoma," Melanoma Research, Vol. 13, No. 4, 2003, pp. 427-429. http://dx.doi.org/10.1097/00008390-200308000-00014

[25] S. Pasquali, A. P. van der Ploeg, S. Mocellin, J. R. Stretch, J. F. Thompson and R. A. Scolyer, "Lymphatic Biomarkers in Primary Melanomas as Predictors of Regional Lymph Node Metastasis and Patient Outcomes," Pigment Cell and Melanoma Research, Vol. 26, No. 3, 2013, pp. 326-337. http://dx.doi.org/10.1111/pcmr.12064

[26] P. P. Aung, M. Sarlomo-Rikala, J. Lasota, J. P. Lai, Z. F. Wang and M. Miettinen, "KBA62 and PNL2: 2 New Melanoma Markers-Immunohistochemical Analysis of 1563 Tumors Including Metastatic, Desmoplastic, and Mucosal Melanomas and Their Mimics," American Journal of Surgical Pathology, Vol. 36, No. 2, 2012, pp. 265272. http://dx.doi.org/10.1097/PAS.0b013e31823651cb

[27] D. P. Kestler, J. S. Foster, C. T. Bruker, J. W. Prenshaw, S. J. Kennel, J. S. Wall, D. T. Weiss and A. Solomon, "ODAM Expression Inhibits Human Breast Cancer Tumorigenesis," Breast Cancer: Basic and Clinical Research (Auck1), Vol. 5, 2011, pp. 73-85.

[28] J. S. Foster, L. M. Fish, J. E. Phipps, C. T. Bruker, J. M. Lewis, J. L. Bell, A. Solomon and D. P. Kestler, "Odontogenic Ameloblast-Associated Protein (ODAM) Inhibits Growth and Migration of Human Melanoma Cells and Elicits PTEN Elevation and INACTIVATION of PI3K/ AKT Signaling," BMC Cancer, Vol. 13, 2013, p. 227. http://dx.doi.org/10.1186/1471-2407-13-227

[29] J. A. Curtin, J. Fridlyand, T. Kageshita, H. N. Patel, K. J.
Busam, H. Kutzner, K. H. Cho, S. Aiba, E. B. Brocker, P. E. LeBoit, D. Pinkel and B. C. Bastian, "Distinct Sets of Genetic Alterations in Melanoma," New England Journal of Medicine, Vol. 353, No. 20, 2005, pp. 2135-2147. http://dx.doi.org/10.1056/NEJMoa050092

[30] H. Tsao, V. Goel, H. Wu, G. Yang and F. G. Haluska, "Genetic Interaction between NRAS and BRAF Mutations and PTEN/MMAC1 Inactivation in Melanoma," Journal of Investigative Dermatology, Vol. 122, No. 2, 2004, pp. 337-341. http://dx.doi.org/10.1046/j.0022-202X.2004.22243.x

[31] L. P. Cantini, F. Meier, V. K. Sondak and K. S. Smalley, "The Future of Targeted Therapy Approaches in Melanoma," Expert Opinion on Drug Discovery, Vol. 4, No. 4, 2009 , pp. 445-456. http://dx.doi.org/10.1517/17460440902828298

[32] W. H. Chappell, L. S. Steelman, J. M. Long, R. C. Kempf, S. L. Abrams, R. A. Franklin, J. Basecke, F. Stivala, M. Donia, P. Fagone, G. Malaponte, M. C. Mazzarino, F. Nicoletti, M. Libra, D. Maksimovic-Ivanic, S. Mijatovic, G. Montalto, M. Cervello, P. Laidler, M. Milella, A. Tafuri, A. Bonati, C. Evangelisti, L. Cocco, A. M. Martelli and J. A. McCubrey, "Ras/Raf/MEK/ERK and PI3K/ PTEN/Akt/mTOR Inhibitors: Rationale and Importance to Inhibiting These Pathways in Human Health," Oncotarget, Vol. 2, No. 3, 2011, pp. 135-164.

[33] J. B. Korman and D. E. Fisher, "Developing Melanoma Therapeutics: Overview and Update," Wiley Interdisciplinary Reviews: Systems Biology and Medicine, Vol. 5, No. 3, 2013, pp. 257-271. http://dx.doi.org/10.1002/wsbm.1210

[34] T. Shimizu, A. W. Tolcher, K. P. Papadopoulos, M. Beeram, D. W. Rasco, L. S. Smith, S. Gunn, L. Smetzer, T. A. Mays, B. Kaiser, M. J. Wick, C. Alvarez, A. Cavazos, G. L. Mangold and A. Patnaik, "The Clinical Effect of the Dual-Targeting Strategy Involving PI3K/AKT/ mTOR and RAS/MEK/ERK Pathways in Patients with Advanced Cancer," Clinical Cancer Research, Vol. 18, No. 8, 2012, pp. 2316-2325. http://dx.doi.org/10.1158/1078-0432.CCR-11-2381

[35] S. J. Vidwans, K. T. Flaherty, D. E. Fisher, J. M. Tenenbaum, M. D. Travers and J. Shrager, "A Melanoma Molecular Disease Model," PLoS One, Vol. 6, No. 3, 2011, Article ID: e18257. http://dx.doi.org/10.1371/journal.pone.0018257 